\title{
Design and construction of, and physics results from an INO RPC detector stack
}

\author{
Gobinda Majumder*E-mail: gobindaetifr.res.in, \\ B. Satyanarayana, V. Datar, K.C. Ravindran \\ Tata Institute of Fundamental Research, Mumbai, \\ S. Pethuraj, E-mail: s.pethuraj@tifr.res. in, Suryanaraya Mondal, Tata Institute of \\ Fundamental Research, Mumbai \& Homi Bhabha National Institute, Mumbai
}

\begin{abstract}
India-based Neutrino Observatory (INO) has proposed a 50 kton magnetised Iron Calorimeter (ICAL) in an underground laboratory to be constructed near Madurai. Main aims of this project are to precisely study the atmospheric neutrino oscillation parameters and to determine the ordering of neutrino masses. The detector will deploy about 28,800 glass Resistive Plate Chambers (RPCs) of approximately $2 \mathrm{~m} \times 2 \mathrm{~m}$ in area. About 3.6 million detector channels are required to be instrumented.

A detector stack comprising of 12 layers of $2 \mathrm{~m} \times 2 \mathrm{~m}$ RPCs was designed and commissioned in Madurai. These RPCs are readout through 60 pickup strips (of pitch $3 \mathrm{~cm}$ ) on the X-plane and 63 on orthogonal Y-plane. The signals which are induced on strips due to passage of charged particles through RPC detectors are processed by a series of electronic elements such as AnalogFront End (AFE), Digital-Front End (DFE) and back-end data acquisition (DAQ) system. On an event trigger mostly caused by passage of atmospheric muon through the detector stack, the coordinates of all strip hits and their timing with reference to the global trigger signal are recorded by the DAQ system. The entire electronics, data acquisition and trigger systems were developed in-house using preamplifier, discriminator and TDC ASICs as well as high-end FPGAs.

Apart from test of long term performance of RPC detectors and electronics, this stack was utilised even to study a few physics problems such as integrated muon flux, polar and azimuthal distribution of incident cosmic ray muons as well as multiplicity of muons passing through the stack.
\end{abstract}

The 39th International Conference on High Energy Physics (ICHEP2018)

4-11 July, 2018

Seoul, Korea

\footnotetext{
* Speaker.

†Corresponding author
} 


\section{Introduction}

The Iron Calorimeter (ICAL) detector in the proposed underground laboratory inside the proposed India-based Neutrino Observatory (INO) at Bodi Hill $\left(9^{\circ} 58^{\prime} N, 77^{\circ} 16^{\prime} E\right)$ will measure atmospheric neutrinos to make a precise measurement of neutrino oscillation parameters including the sign of the 2-3 mass-squared difference, $\Delta m_{32}^{2}\left(=m_{3}^{2}-m_{2}^{2}\right)$ using the matter effect. A detailed description of the INO project can be found in [1]. The Resistive Plate Chamber (RPC) [2, 3] is chosen as the sensitive detector element in the ICAL detector due to its excellent time resolution and low cost. To study the long term stability of RPC produced in Indian industry, a stack of 12 layer RPC is setup at transit campus of IICHEP, Madurai, which was operational for three years and performances of that stack provides a huge input toward construction of the RPC and related electronics for the final ICAL experiment.

\section{Electronics}

The RPCs are operated in the avalanche mode with muon detection efficiencies in the central region of $(95.0 \pm 0.2) \%$ at an applied voltage of $10.0 \mathrm{kV}( \pm 5.0 \mathrm{kV})$ across the gas gaps. The experimental setup with an isolated RPC with complete electronics is shown in figure 1.
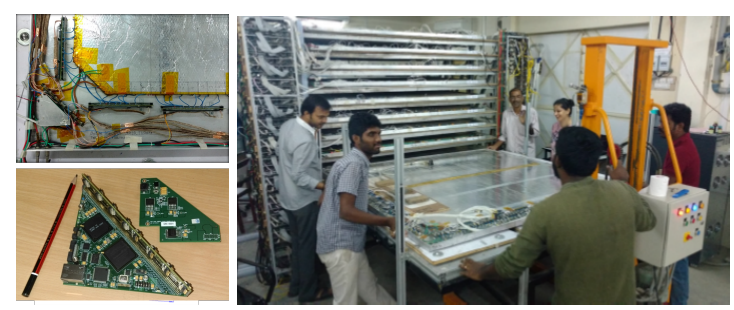

Figure 1: The 12 layer RPC stack and its components at IICHEP campus, Madurai.

The induced RPC signal which is picked up by the readout strip is fed to analog front-end boards, which is designed with 4-channel voltage amplifier and 8-channel leading edge discriminator ASIC. These data is processed through the electronics module, call digital back-end (DFE, left in figure 1), which comprises of several functional blocks such as a Time-to-Digital converter, strip-hit latch, rate monitor, pre-trigger generator, ambient parameter monitor and Front-end control. The An event data typically comprises of hit pattern of strips (one logic bit per strip) from Xand Y-planes as well as eight timing informations of both plane. This study is based on the trigger criteria, where at least one strip in Layer-4,5,6\&7 have signal more than $V_{t h}(=-20 \mathrm{mV})$ within a time window of $100 \mathrm{~ns}$, where RPC number is starting from the bottom.

\section{Performance}

Typical multiplicity of RPC strips are shown in figure 2. Multiplicity is peaked in 1-2, which is expected due to a trajectory of muon. Variation of multiplicity in those two chambers are mainly due to variation of gain in those chambers. Though the peak of multiplicity lies near one, it has a tail upto maximum strips (60/63 for X/Y-side). Sources of different multiplicities are categories as (i) no hit implies inefficiency, (ii) 1 to 4 hits are due avalanche signal in RPC, (iii) 5-20 hits are due to streamer mode signal and $(i v)$ others are mainly due to correlated electronics noise. 
The position dependent efficiency, timing performance etc of an RPC detector is estimated by fitting $2 \mathrm{D}$ muon trajectories in the $\mathrm{XZ}$ and $\mathrm{YZ}$ plans. The performance of each layer is estimated by excluding that layer in the straight line fit of the muon trajectory and hit multiplicity in the used layer were kept inbetween 1 to 3 .
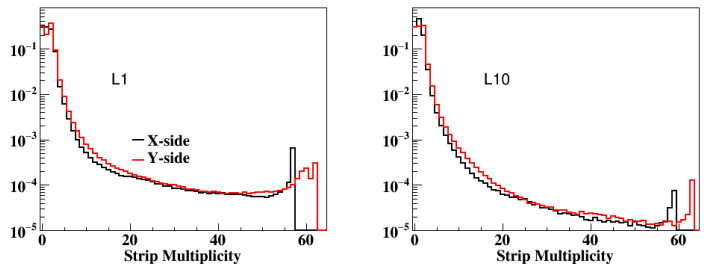

Figure 2: Strip multiplicity in layer-1 and layer-10.

An offline alignment corrections derived from the muon trajectories give an overall position accuracy of better than $100 \mu \mathrm{m}$. Similarly channel-wise time correction are obtained using iterative method of offset correction [4].

\section{Position dependent efficiency and strip multiplicity as a function of muon position in a strip}

The muon detection efficiency in layer-1 and layer-2 are shown in figure 3. strip. Grid like inefficiency points are due to button spacer, inefficiency along a strip is due to dead electronics and low efficiency in a region is due to improper graphite coating and/or large gap inside RPC.

Induced signal in RPC strips depends on the position of muon trajectory in strips local coordinate system. Muon detection efficiency as a function of muon trajectory in the strip co-ordinate is shown in figure 4 . The probability of having only one/two strip hits for the muon trajectory at the center/edge of the strip is due to induced charge sharing in neighbouring strips.
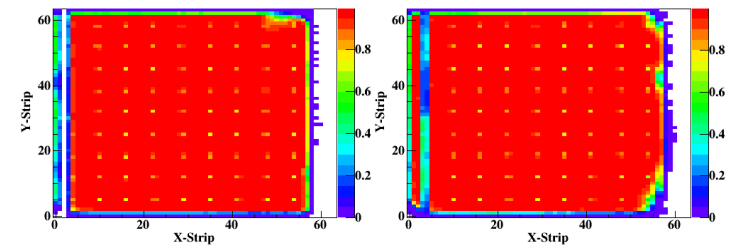

Figure 4: Efficiency of muon trajectory (left) Figure 3: Efficiency of muon hits in various posi- Layer-2 and (right) layer-4 as a function of positron tion in (left) Layer-1 and (right) layer-2.
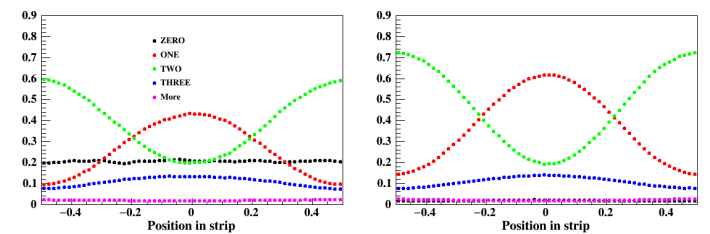

\section{Time resolution and directionality}

Typical time resolution of the RPC stack is shown in left plot in figure 5, which was within our expectation. Measurement of muon velocity is shown in tight plot in figure 5, where $\beta=1(-1)$ implies down-going (up-going) muon with velocity $\sim c$. Thus the estimated mistag rate of up-going muon (with $-\mathrm{ve} \beta$ ) is only one in $10^{5}$ events and this performance was in our design goal.

\section{Azimuthal angle of cosmic muon}

The CORSIKA simulation package [5] is use to generate muon spectrum at IICHEP site, the Geant 4 tool-kits is used to propagate muon trajectory in the detector setup and reconstructed muon 

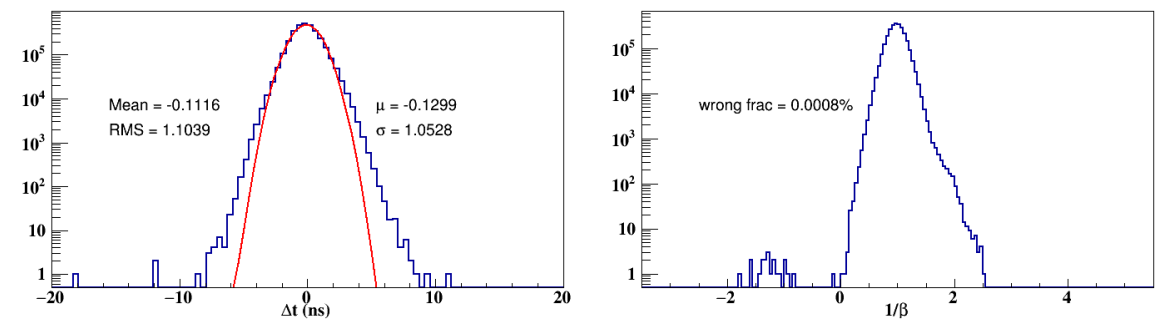

Figure 5: (left) Time resolution of RPC in Layer-9 for different multiplicity and (right) Measured velocity of cosmic muon.

spectrum is compared with the observed spectrum. Figure 6 shows that the data is matched well in most of the azimuthal region except from "North" direction and this discrepancies increases with larger zenith angle, but the difference is independent of hadronisation models in CORSIKA.
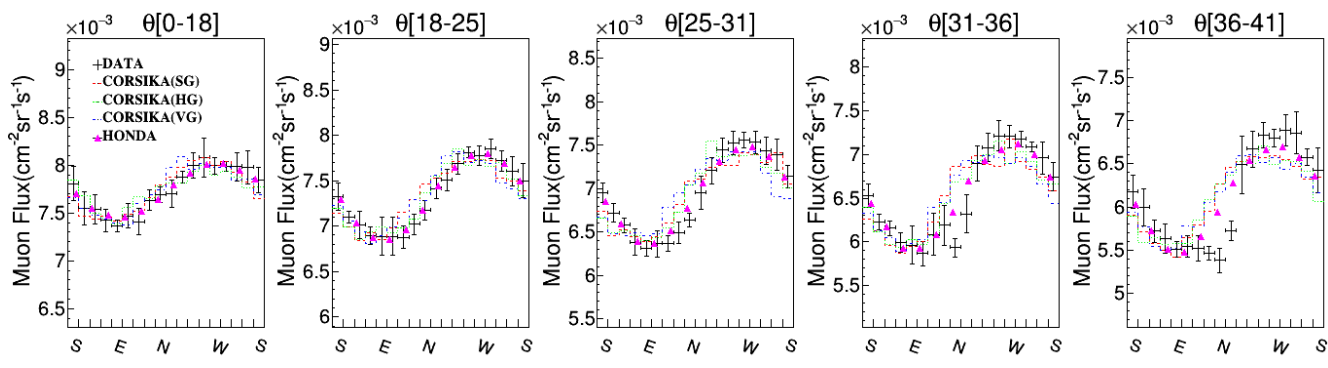

Figure 6: Comparison of azimuthal angle distribution of data with CORSIKA simulation.

\section{Summary}

We had successfully tested twelve RPC detector of size $2 \mathrm{~m} \times 2 \mathrm{~m}$ for more than three years at Madurai. Efficiency of RPC detector were from $90-95 \%$ and in different detector and with small variation with local position of the RPC. Timing resolution of the detector is about $1 \mathrm{~ns}$. Using this setup, we have found that up-down ambiguity of muon is negligible and obtained azimuthal angle of muon is compared with the prediction of CORSIKA simulation.

\section{References}

[1] Ahmed, Shakeel and others Physics Potential of the ICAL detector at the India-based Neutrino Observatory (INO), Pramana 88 (2017) no.5, 79

[2] Pestov, Yu. N. and Fedotovich, G. V. a picosecond time-of-flight spectrometer for the vepp-2m based on local - discharge spark counter, SLAC-TRANS-0184 IYF-77-78 (1978).

[3] R. Santonico and R. Cardarelli, Development of resistive plate counters, Nucl. Instrum. Methods. 187 (1981) 377.

[4] G. Majumder, N.K. Mondal, S. Pal, D. Samuel, B. Satyanarayana, Study of the directionality of cosmic muons using the INO-ICAL prototype detector, Nucl.Instrum.Meth A735 (2014) 88-93.

[5] D.Heck, J.Knapp, J.N.Capdevielle, G.Schatz, and T.Thouw, Report FZKA 6019 (1998), Forschungszentrum Karlsruhe, https://www.ikp.kit.edu/corsika/70.php 